\title{
Association analysis of human leukocyte antigen class II (DRB1) alleles with leprosy in individuals from São Luís, state of Maranhão, Brazil
}

\author{
Rita da Graça Carvalhal Frazão Corrêa ${ }^{1 /}{ }^{+}$, Dorlene Maria Cardoso de Aquino ${ }^{1}$, \\ Arlene de Jesus Mendes Caldas ${ }^{1}$, Humberto de Oliveira Serra', \\ Fábio França Silva ${ }^{2}$, Maxwellem de Jesus Costa Ferreira², \\ Elton Jonh Freitas Santos ${ }^{2}$, Emygdia Rosa Rêgo Barros Pires Leal Mesquita ${ }^{3}$
}

\author{
${ }^{1}$ Grupo de Pesquisa em Epidemiologia de Doenças Transmissíveis ${ }^{2}$ Hospital Universitário ${ }^{3}$ Laboratório de Estudos \\ Genômicos e Histocompatibilidade, Universidade Federal do Maranhão, São Luís, MA, Brasil
}

\begin{abstract}
Epidemiological studies have demonstrated that the variability of the clinical response to infection caused by Mycobacterium leprae is associated with host genetic factors. The present study investigated the frequency of human leukocyte antigen (HLA) class II (DRB1) alleles in patients with leprosy from São Luís, Maranhão, Brazil. A case-control study was performed in 85 individuals with leprosy and 85 healthy subjects. All samples were analysed via polymerase chain reaction-sequence specific oligonucleotide probes. The HLA-DRBI*16 allele showed a higher frequency in the group with leprosy $[(9.41 \%$ vs. $4.12 \%)$ odds ratio $(O R)=2.4195 \%$ confidence interval (CI) (0.966.08) $p=0.05]$, whereas the HLA-DRB1*11 allele was less frequent in the group with leprosy [(6.47\% vs. $11.76 \%)$ OR $=0.5195 \%$ CI (0.23-1.12) $p=0.09]$. The frequency of HLA-DRBI* alleles between the control group and leprosy patient subgroups presenting different forms of the disease showed that the HLA-DRB1*16 $(16.13 \% \mathrm{vs} .8 .24 \%$, OR $=4.10, C I=1.27-13.27, p=0.010)$ and $H L A-D R B 1 * 14(5 \% v s .3 .53 \%, O R=4.63, C I=1.00-21.08, p=0.032)$ alleles were significantly more frequent in patients with different clinical subtypes of leprosy. The sample size was a limitation in this study. Nevertheless, the results demonstrated the existence of a genetic susceptibility associated with the clinical forms of leprosy. The low frequency of the HLA-DRB1*11 allele should be further studied to investigate the possible protective effect of this allele.
\end{abstract}

Key words: leprosy - HLA-DRB1 gene - association study

Leprosy is an infectious disease caused by Mycobacterium leprae, which is an intracellular parasite that reproduces slowly inside phagocytic cells and has a predilection for cutaneous tissue and the peripheral nervous system. M. leprae constitutes a serious social and public health problem due to the physical deformities and disabilities that it causes, leading to discrimination and segregation of patients (Virmond 2008, Walter \& Lockwood 2007).

According to the World Health Organization (2010) statistics, leprosy had a registered prevalence of 192,246 cases worldwide and 228,474 new cases were detected. Among the 130 countries in which this disease is considered endemic, only 17 accounted for $95 \%$ of the new cases detected worldwide. In Brazil, the recorded prevalence was 29,761 and the number of new cases was 34,894 , including only 14,263 cases of multibacillary (MB). Among the new cases, the number of females was 15,513 and the number of children was 2,461 . The number of new cases with grade 2 disabilities was 2,241.

Financial support: LEGH/HUUFMA

+ Corresponding author: profaritacarvalhal@gmail.com

Received 12 February 2012

Accepted 25 July 2012
In 2010, Brazil did not register any cases of relapse and there was an $85 \%$ cure rate for paucibacillary (PB) and an $80 \%$ cure rate for MB cases.

Although the incidence of leprosy in the world continues to diminish, an on-going effort at the international level is still required to ensure that the number of cases continues to decrease in countries in which the disease is endemic. With this intention, a new global initiative was adopted by the WHO (2009). The initiative seeks to reduce the number of new cases with grade 2 disabilities per 100,000 individuals by at least $35 \%$ by the end of 2015. This decrease corresponds to a $44 \%$ reduction in the prevalence rate of leprosy by 2015, from $1.75-0.98 / 10,000$ individuals, starting from the prevalence numbers recorded at the end of 2010 (WHO 2011).

Leprosy is considered to be a complex multifactorial disease. Studies have demonstrated the important role of host genes that influence the immune response against $M$. leprae. Only approximately $1 \%$ of individuals exposed to the bacillus become sick, supporting the hypothesis that genetic factors play a role and highlighting the influence of human leukocyte antigen (HLA) alleles (Moraes et al. 2006, Virmond 2008, Francheschi et al. 2009).

The HLA complex is being studied in leprosy patients (LPs) because of the direct involvement of these alleles in the immune response. Investigating HLA genes could therefore elucidate the mechanisms of susceptibility or resistance to the disease (Goulart \& Goulart 2009). 
Association studies and comparisons of allele frequencies have been employed to determine the host molecular characteristics associated with M. leprae infections. These studies have shown that different genes in particular play an important role in the activation of immune responses and that HLA alleles influence not only the susceptibility or resistance to the disease, but also the disease course in terms of both infection control and the manifestation of the different clinical forms (Alves et al. 2007, Virmond 2008, Francheschi et al. 2009).

The main function of HLA molecules is to present peptides derived from M. leprae to host T-cells. Susceptibility to an infectious disease may be associated with defects in some aspect of this system. An individual who has a particular combination of HLA alleles that are not able to associate with the peptide in an appropriate way or in whom the HLA-peptide linkage does not properly activate lymphocytes will be more susceptible to the infectious agent than an individual who does not exhibit such defects (Klein \& Sato 2000). In patients whose HLA systems offer protection against a disease, these genes will be able to properly stimulate T-cells, which will expand and eliminate the agent by producing inflammatory cytokines, which destroy infected cells (Alves et al. 2005, Mira 2006).

According to Ridley and Jopling (1966), the clinical manifestation of leprosy depends on the type of immune response that is initiated by the host and the balance between T-helper (Th)1 and Th2 responses may be partially controlled by the mechanism of antigen presentation involving HLA molecules (Mira 2006, Singh et al. 2007). The tuberculoid (TT) form of leprosy is associated with a Th1 (cellular) immune response, characterised by the production of proinflammatory cytokines that can participate in the clearance of the bacillus. However, the lepromatous (LL) form of leprosy is associated with a Th2 (humoral) immune response, which is characterised by an immunosuppressive cytokine environment, making this type of response problematic for the host. The TT and LL forms correspond approximately to the PB and MB forms, respectively, of leprosy defined by the WHO for therapeutic purposes (Ridley \& Jopling 1966, MS/FNS 1994, Vanderborght et al. 2007).

Prior to the discovery of the causative agent of leprosy in 1874 by Armauer Hansen, it was believed that this disease was hereditary (Ridley \& Jopling 1966, Jambeiro et al. 2008). Subsequently, data from studies on families and twins, combined with the fact that only a small proportion of infected individuals become sick and that the disease prevalence is dependent on ethnicity, suggested a genetic susceptibility component to the disease (Beiguelman 2002). Thus, increased attention is now being paid to the major histocompatibility complex (MHC). In humans, the MHC is referred to as the HLA and was first described by Dausset (1958), in France. The HLA comprises a region of highly polymorphic genes located on the short arm of chromosome 6 (6p21). Each locus may contain one of many genes encoding a specific glycoprotein, allowing for wide variation in the expression of these glycoproteins and modulation of immune responses that determine the form of the disease (Benjamini et al. 2002, Monte et al. 2004, Alves et al. 2005, 2007).

Current studies are therefore seeking to elucidate the genetic mechanisms of susceptibility or resistance to leprosy. Positive associations between the TT form and HLA-DR alleles and between the LL form and HLA-DQ alleles have been described (Visentainer et al. 1997, Alves et al. 2006, Moraes et al. 2006, Souza \& Pereira 2007).

Other population studies conducted in many parts of the world have also shown an association between the TT and LL forms of the disease and HLA class II loci. Based on these findings, Vanderborght et al. (2007) investigated the influence of the HLA-DRB1 locus on susceptibility to leprosy and showed that HLA-DRB1*10 was associated with susceptibility, while HLA-DRB1*04 was associated with resistance to leprosy in both Brazilian and Vietnamese populations. These results suggest that class II alleles play an important role in the activation of cellular immune responses against $M$. leprae.

According to Souza and Pereira (2007), the current understanding of the molecular basis of the disease and the continuous surveillance of leprosy in Brazil suggest that the admixture of races in the country may have generated a genetic profile that could contribute to the occurrence of leprosy. Hence, epidemiologic studies focusing on genetics in the Brazilian population are extremely relevant.

Finally, studies investigating leprosy in admixed groups are important because these studies permit the identification of new associations or confirm previously described associations. The type of association detected might vary with the ethnic-racial group studied. The objective of the present study was to determine the frequency of HLA class II (DRB1) alleles and their association with leprosy in individuals from São Luís, Maranhão (MA), in northeastern Brazil.

\section{SUBJECTS, MATERIALS AND METHODS}

Patients and controls - The study described herein was a case-control study performed using individuals with leprosy and was carried out with the assistance of the Leprosy Control Program of the University Hospital of the Federal University of Maranhão (HUUFMA), São Luís. Samples were collected from leprosy carriers with an active record, who were identified by searching the register of new cases and using individual notification forms.

We selected 85 patients, independent of the clinical form of the disease they presented. To define the study groups, we adopted the classification of Madrid (1953) that follows the criterion of polarity, which is based on the clinical characteristics of the disease as well as the bacteriological, immunological and histological features of leprosy. We defined the polar groups as exhibiting the TT, virchowian or LL form, the transitional or initial group as exhibiting an indeterminate form and the unstable intermediate group as exhibiting a borderline (BB) or dimorphous form (WHO 1989). The clinical cases consisted of 20 patients with the TT form, 42 patients with the LL form, 26 patients with the BB form and tree patients with the indeterminate form. 
The control group was composed of healthy unrelated individuals who were randomly recruited from the same geographic region where the last case arose. The control individuals had no history of HLA-associated diseases (infectious or autoimmune diseases). The risk of bias between the allele frequencies was minimised by matching cases and controls with the same ethnic origin, mean age and sex that came from the same geographical area.

Demographic data were collected using forms that included epidemiological and clinical data, as well as medical records and reporting forms. The collection of biological material was performed after an interview at the clinic or at home after contact and scheduling. The samples were genotyped by the Laboratory of Genetic Studies and Histocompatibility, HUUFMA. All participants signed a consent form authorising the use of their samples in the study. This study was approved by the Research Ethics Committee of the University Hospital, protocol 0016/2008.

Extraction of genomic DNA - Blood samples $(8 \mathrm{~mL})$ were collected in vacutainer tubes containing an anticoagulant (ethylenediamine tetraacetic acid) and centrifuged at $1,500 \mathrm{rpm}$ for $15 \mathrm{~min}$. The buffy coat was collected and stored at $-20^{\circ} \mathrm{C}$ until use. DNA was extracted using EZ-DNA ${ }^{\circledR}$ kits (Biological Industries, Kibbutz Beit Haemek, Israel) from $500 \mu \mathrm{L}$ of the buffy coat according to the manufacturer's protocol.

Determination of HLA class II alleles - Amplification of DNA fragments corresponding to HLA class II alleles was performed via polymerase chain reaction-sequence specific oligonucleotide (PCR-SSO) using RSSO2B1 primers for the HLA class II DRB1 locus and the Lab Type ${ }^{\mathrm{TM}}$ SSO kit (One Lambda ${ }^{\circledR}$ Inc, Canoga Park, CA, USA). The samples were processed in a Labscan ${ }^{\mathrm{TM}} 100$ flow cytometer and analysed using the HLA Fusion ${ }^{\mathrm{TM}}$ program (One Lambda, Canoga Park, CA, USA, version 2.0.0).

The obtained PCR products were analysed via gel electrophoresis in a $2 \%$ agarose gel and stained with a $0.05 \%$ ethidium bromide solution for $4 \mathrm{~min}$. The gel was visualised under ultraviolet transillumination and photo documentation was performed using an automated system (Kodak Digital Science - Electrophoresis Documentation and Analysis System 120, Eastman Kodak Company, NY, USA).

Statistical analysis - The allele frequencies in the two groups were compared using the chi-square test or Fisher's exact test when necessary. To estimate risk, the odds ratio (OR) was calculated with a $95 \%$ confidence interval (CI). The significance level considered was $5 \%$ $(p<0.05)$. The data were processed and analysed using Stata 10.0 (Stata Corporation, College Station, Texas, USA) and Epi Info ${ }^{\mathrm{TM}}$ version 3.4.3 software (Centers for Disease Control and Prevention, Atlanta, USA).

Allele frequencies were determined through direct counting of the alleles. The distributions were confirmed to follow Hardy-Weinberg equilibrium by calculating the expected frequencies of genotypes and comparing them with the observed values using Arlequin version 3.1.1 software (Zoological Institute, University of Berne, Switzerland) (Excoffier et al. 2005).

\section{RESULTS}

In this study, the likely association between HLA class II alleles and leprosy in individuals from São Luís was investigated by comparing the frequencies of HLA-DRB1 alleles between leprosy carriers and healthy controls (HCs).

General characteristics of the study groups - The study involved 170 individuals, 85 of whom were LPs, while 85 were healthy individuals. The leprosy carriers were classified as follows: $23.5 \%$ TT, $42.5 \% \mathrm{LL}, 30.5 \%$ $\mathrm{BB}$ and $3.5 \%$ indeterminate. At the time of sample collection, the ages of the patients ranged between 10-79 years $(51.8 \%$ males and $48.2 \%$ females). In the control group, $32.9 \%$ were male and $67.1 \%$ were female, with ages ranging between eight-70 years. With respect to skin colour, $60 \%$ of the LPs were reported to exhibit a brown skin colour and $25.9 \%$ to present a black skin colour. Concerning the control individuals, $69.4 \%$ were reported to have a brown skin colour and $23.5 \%$ to present a black skin colour (Table I).

$L P S$ and control group - In all of the groups, the distribution of HLA-DRB1* genotypes was confirmed to be in Hardy-Weinberg equilibrium ( $p=0.09$ in the patients and $\mathrm{p}=0.45$ in the controls).

Analysis of the allele frequencies in the patients with leprosy and the control group for the HLA-DRB1 locus detected the presence of 13 of the 975 alleles described in the literature (The International Immunogenetics Information System ${ }^{\circledR}$ ) (ebi.ac.uk/imgt/hla/stats.html), which is equivalent to $1.33 \%$ of the total reported alleles.

\section{TABLE I}

Leprosy carriers according to sex, skin colour, age and clinical form, São Luís, state of Maranhão, Brazil, 2010

\begin{tabular}{lcc}
\hline Variable & $n(\%)$ & $95 \%$ CI \\
\hline Sex & & \\
$\quad$ Male & $44(51.8)$ & $40.7-62.7$ \\
Female & $41(48.2)$ & $37.3-59.3$ \\
Skin colour & & \\
$\quad$ White & $12(14.1)$ & $7.5-23.4$ \\
Black & $20(25.9)$ & $15.0-34.0$ \\
Brown & $53(60)$ & $51.2-72.6$ \\
Age & & \\
$\quad<15$ & $4(4.7)$ & $1.3-11.6$ \\
$15-19$ & $7(8.2)$ & $3.4-16.2$ \\
$20-39$ & $34(40)$ & $29.5-51.2$ \\
$40-64$ & $35(41.2)$ & $30.6-52.4$ \\
65 or more & $5(5.9)$ & $1.9-13.2$ \\
Clinical form & & \\
Indeterminate & $3(3.5)$ & $1.3-11.6$ \\
Tuberculoid & $20(23.5)$ & $15.0-34.0$ \\
Lepromatous & $36(42.5)$ & $25.2-46.4$ \\
Borderline & $26(30.5)$ & $26.3-47.6$ \\
\hline Total & $85(100)$ & - \\
\hline CI: confidence & & \\
\hline
\end{tabular}

CI: confidence interval. 
HLA-DRB1*16 and DRB1*14 showed a higher frequency in the leprosy group compared with the control group $(9.41 \%$ vs. $4.12 \%, \mathrm{OR}=2.41, \mathrm{CI}=0.96-6.08, \mathrm{p}=$ 0.05 and $5.29 \%$ vs. $1.76 \%, \mathrm{OR}=3.11, \mathrm{CI}=0.82-11.79$, $\mathrm{p}=0.07$, respectively). Although these results did not present statistically significant differences, the HLADRB1*14 and DRB1*16 alleles exhibited a trend toward an association with the disease. HLA-DRB1*11 was less frequent in the leprosy group compared with the control group, suggesting a potential protective effect of HLADRB1 $* 11$ against leprosy $(6.47 \%$ vs. $11.76 \%, \mathrm{OR}=0.51$, $\mathrm{CI}=0.23-1.12, \mathrm{p}=0.09)$ (Table II).

The frequencies of HLA-DRB1 alleles were also different between the control group and the patient subgroups representing different forms of the disease. The HLA-DRB1*16 allele was associated with the LL clinical form $(16.13 \%$ vs. $8.24 \%$, OR $=4.10, \mathrm{CI}=1.27-13.27$, $\mathrm{p}=0.010$ ), whereas the HLA-DRB1*14 allele showed a higher frequency in the TT group $(5 \%$ vs. $3.53 \%$, OR = $4.63, \mathrm{CI}=1.00-21.08, \mathrm{p}=0.032)$, thus demonstrating an association (Table III).

When the allele frequencies were compared between the TT and LL clinical forms, no statistically significant difference was observed (data not shown).

\section{DISCUSSION}

This study investigated the association between the frequency of HLA-DRB1 alleles in a group of LPs in comparison with HCs from São Luís.

Association studies and comparisons of allele frequencies have demonstrated the involvement of HLA class II as a factor associated with host responses to infection with M. leprae. The results of these studies have indicated that HLA genes play an important role in the activation of immune responses. HLA alleles influence not only the susceptibility or resistance to the disease, but also the disease course in terms of both control of the infection and the development of different clinical forms of leprosy (Mira 2006, Alves et al. 2007, Virmond 2008, Francheschi et al. 2009).

In the present study, the HLA-DRB1*16 allele was found to present a higher frequency in the leprosy group compared with the control group, indicating a tendency toward disease susceptibility in the analysed samples. In Brazil, associations between HLA-DRB1*16 and leprosy have been detected in analyses performed by Silva et al. (2009), who studied a population in the state of Paraná, southern Brazil. This allele is a susceptibility marker for leprosy per se, confirming the results of a previous study that showed an association between HLA-DR2 and leprosy in patients from an equivalent geographic region (Visentainer et al. 1997). An association of HLA-DRB1*16 with susceptibility to leprosy per se has been described in many populations around the world, such as in Suriname, Brazil, Venezuela, Egypt, China, Japan, Korea, Taiwan, Mexico and Turkey (Hegazy et al. 2002, Joko et al. 2000, Gorodezky et al. 2004, Hsieh et al. 2010).

Genetic analysis of a large sample of Chinese LPs provided evidence of an association of HLA-DRB1*15 with susceptibility and of HLA-DRB1*09 with protection (Zhang et al. 2009). Other studies have demonstrated that the DRB1*1501 allele is more frequent among Euro-descendants, whereas the DRB1*1503 allele is found more often in Afro-descendants (Moraes et al. 2006).

In the study performed by Vanderborght et al. (2007), the HLA-DRB ${ }^{*} 04$ allele was associated with protection against leprosy, whereas the HLA-DRB1*10 allele was associated with susceptibility. The large sample size investigated in the last study allowed the observation to be made that there was an ethnicity-specific association in the Brazilian population, as the HLA-DRB1*10 allele was found to be highly associated with susceptibility to leprosy exclusively in the group stratified as African-Brazilians.

In our analysis of the association of the HLA-DRB1* locus with leprosy, the HLA-DRB1*14 $(\mathrm{OR}=3.11)$ and HLA-DRB1*16 $(\mathrm{OR}=2.41)$ alleles presented a stronger association (3 and 2 times more, respectively) with patients affected with the disease. However, these results were not statistically significant. The identification of HLA genes that are associated with diseases in highly admixed populations, such as the Brazilian population, may be difficult because of the great variety of polymorphisms present. However, this kind of study is important because it allows the discovery of new associations as well as the confirmation of associations described previously (Donadi 2000, Alves et al. 2006).

The HLA-DRB1*11 allele $(\mathrm{OR}=0.51)$ exhibited a two-fold lower frequency in children with leprosy; however, this difference was not statistically significant.

\section{TABLE II}

Allele frequency for the human leukocyte antigen-DRB1 locus in leprosy patients vs. control group, São Luís, state of Maranhão, Brazil, 2010

\begin{tabular}{|c|c|c|c|c|}
\hline & $\begin{array}{l}\text { Leprosy } \\
(\mathrm{n}=85)\end{array}$ & $\begin{array}{l}\text { Control } \\
(\mathrm{n}=85)\end{array}$ & & \\
\hline Alleles & n $(\%)$ & n $(\%)$ & $\mathrm{p}$ & OR $(95 \% \mathrm{CI})$ \\
\hline DRB1*01 & $16(9.41)$ & $17(10)$ & 0.85 & $0.93(0.45-1.92)$ \\
\hline DRB1*03 & $12(7.06)$ & $17(10)$ & 0.33 & $0.68(0.31-1.48)$ \\
\hline DRB1*04 & $12(7.06)$ & $14(8.23)$ & 0.68 & $0.84(0.37-1.89)$ \\
\hline DRB1*07 & $12(7.06)$ & $8(4.73)$ & 0.35 & $1.53(0.61-3.87)$ \\
\hline DRB1*08 & $25(14.70)$ & $20(11.76)$ & 042 & $1.29(0.68-2.43)$ \\
\hline DRB1*09 & $11(6.47)$ & $10(5.88)$ & 0.82 & $1.10(0.45-2.68)$ \\
\hline DRB1*10 & $6(3.56)$ & $4(2.35)$ & 0.52 & $1.51(0.41-5.49)$ \\
\hline $\mathrm{DRB} 1 * 11^{a}$ & $11(6.47)$ & 20 (11.76) & 0.09 & $0.51(0.23-1.12)$ \\
\hline DRB1*12 & $2(1.17)$ & $5(2.94)$ & 0.25 & $0.39(0.07-2.05)$ \\
\hline DRB1*13 & $16(9.41)$ & $22(12.94)$ & 0.30 & $0.69(0.35-1.38)$ \\
\hline DRB1*14 $4^{b}$ & $9(5.29)$ & $3(1.76)$ & 0.07 & $3.11(0.82-11.79)$ \\
\hline DRB1*15 & $22(12.94)$ & $23(13.53)$ & 0.87 & $0.95(0.50-1.78)$ \\
\hline $\mathrm{DRB} 1 * 16^{c}$ & $16(9.41)$ & $7(4.12)$ & 0.05 & $2.41(0.96-6.08)$ \\
\hline
\end{tabular}

$a$ : lower frequency in the group with leprosy with no statistical significance; $b$ : higher frequency in the group with leprosy with no statistical significance; $c$ : higher frequency in the group with leprosy with trend toward association; CI: confidence interval; OR: odds ratio. 
A different result was obtained by Lavado-Valenzuela et al. (2011) in a study performed in Goiânia, state of Goiás, Brazil. This group found an increased frequency of HLA-DRB1*11 in LL patients compared with HCs, suggesting that the DRB1*11 allele could be associated with susceptibility to LL leprosy.

The clinical forms of leprosy have been widely studied and represent different manifestations of the disease that are dependent on the host immune response. The initiation of Th1 and Th2 responses can be partially controlled by the mechanism of antigen presentation involving HLA molecules (Mira 2006). In the TT form of the disease, the production of proinflammatory cytokines by Th1 cells may assist in the clearance of M. leprae.

\section{TABLE III}

Frequency of human leukocyte antigen class II alleles and clinical forms of leprosy, São Luís, state of Maranhão, Brazil, 2010

\begin{tabular}{|c|c|c|c|c|c|}
\hline Alleles $^{a}$ & n $(\%)$ & $\mathrm{n}(\%)$ & $\mathrm{p}$ & OR & $(95 \% \mathrm{CI})$ \\
\hline & $\begin{array}{l}\text { Lepromatous } \\
\qquad(\mathrm{n}=36)\end{array}$ & $\begin{array}{l}\text { Controls } \\
(\mathrm{n}=85)\end{array}$ & & & \\
\hline $\mathrm{DRB} 1 * 01$ & $7(11.26)$ & $17(20)$ & 0.3221 & - & - \\
\hline $\mathrm{DRB} 1 * 07$ & $5(8.06)$ & $8(9.41)$ & 0.1957 & - & - \\
\hline DRB1*10 & $3(4.84)$ & $4(4.71)$ & 0.3673 & - & - \\
\hline DRB1*11 & $7(11.29)$ & $20(23.53)$ & 0.2091 & - & - \\
\hline DRB1*14 & $3(4.84)$ & $3(3.53)$ & 0.2282 & - & - \\
\hline \multirow[t]{2}{*}{ DRB1*16 } & $10(16.13)$ & $7(8.24)$ & 0.0105 & 4.10 & $1.27-13.27$ \\
\hline & $\begin{array}{l}\text { Borderline } \\
(\mathrm{n}=26)\end{array}$ & $\begin{array}{l}\text { Controls } \\
(\mathrm{n}=85)\end{array}$ & & & \\
\hline DRB1*03 & $4(7.69)$ & $17(20)$ & 0.132 & - & - \\
\hline DRB1*08 & $10(19.23)$ & $20(23.53)$ & 0.872 & - & - \\
\hline $\mathrm{DRB} 1 * 10$ & $1(1.92)$ & $4(4.71)$ & 0.442 & - & - \\
\hline DRB1*14 & 4 (7.69) & $3(3.53)$ & 0.272 & - & - \\
\hline \multirow[t]{2}{*}{ DRB1*15 } & $8(15.38)$ & $23(27.06)$ & 0.607 & - & - \\
\hline & $\begin{array}{l}\text { Tuberculoid } \\
(\mathrm{n}=20)\end{array}$ & $\begin{array}{l}\text { Controls } \\
(\mathrm{n}=85)\end{array}$ & & & \\
\hline DRB1*01 & $2(5)$ & $17(20)$ & 0.482 & - & - \\
\hline $\mathrm{DRB} 1 * 08$ & $4(10)$ & $20(23.53)$ & 0.169 & - & - \\
\hline DRB1*09 & $2(5)$ & 10 (11.76) & 0.168 & - & - \\
\hline DRB1*11 & $2(5)$ & $20(23.53)$ & 0.095 & - & - \\
\hline \multirow[t]{2}{*}{ DRB1*14 } & $2(5)$ & $3(3.53)$ & 0.032 & 4.63 & $(1.00-21.08)$ \\
\hline & $\begin{array}{l}\text { Indeterminate } \\
\qquad(\mathrm{n}=3)\end{array}$ & $\begin{array}{c}\text { Controls } \\
(\mathrm{n}=85)\end{array}$ & & & \\
\hline DRB $1 * 08$ & $2(33.33)$ & $20(23.53)$ & 0.117 & - & - \\
\hline DRB1*13 & $2(33.33)$ & $22(25.88)$ & 0.153 & - & - \\
\hline DRB1*15 & $2(33.33)$ & $23(27.06)$ & 0.173 & - & - \\
\hline
\end{tabular}

$a$ : alleles with higher frequencies in the case group and control group; CI: confidence interval; OR: odds ratio.
However, in the LL form, the immunosuppressive cytokines produced by $\mathrm{Th} 2$ cells hinder a proper response (Mira 2006, Singh et al. 2007). In this regard, studies carried out in different populations around the world have shown positive associations between TT leprosy and HLA-DR2 and DR3 and between LL leprosy and HLA-DQ1 (Singh et al. 2007).

In a study addressing Brazilian and Vietnamese populations, Vanderborght et al. (2007) found an association between the HLA-DRB1* locus and leprosy per se, but not with the clinical subtypes of the disease.

The frequencies of HLA-DRB1 alleles in patients with different subtypes of the disease showed significant differences. The HLA-DRB1*16 allele was associated with the LL clinical form and the HLA-DRB1*14 allele was associated with the TT form. These findings demonstrated a significant association when compared with the results shown in Table II (frequency of alleles between LPs and the control group). Even when the results did not show statistical significance, they indicated trends associated with susceptibility.

The findings described herein differ from those of other studies, such as that by Silva et al. (2009), who found that the HLA-DRB1*04 allele was associated with protection against LL leprosy at a lower frequency than in the group with TT leprosy. A similar result was found in a study involving 89 LPs from the province of Chaco, Argentina, where the HLA-DRB1*04 allele was observed to be present at significantly lower frequency in PB LPs when compared with $\mathrm{HCs}$, suggesting HLA-DRB1*04 to be a protective factor. According to the authors of this paper, the differences between their findings and those of other studies are due to the fact that the inhabitants of Chaco include a considerable admixture of South American natives (Guarani and Tobas) (Motta et al. 2007).

Studies investigating population genetics indicate that admixed groups may show a genetic profile that favours the occurrence of leprosy. Hence, obtaining a better understanding of the impact of genetics in leprosy may lead to the development of new strategies for the diagnosis, prevention and treatment of leprosy in the future (Prevedello \& Mira 2007, Souza \& Pereira 2007).

The results of the present study confirm the significant influence of the frequency of HLA-DRB1 alleles in patient subgroups presenting various forms of the disease. The HLA-DRB1*16 allele was associated with the clinical LL form, whereas the HLA-DRB1*14 allele was associated with the TT form. The sample size and the lack of a test for p-value adjustment may represent limitations of this study. Nevertheless, the results indicated the existence of a genetic susceptibility associated with the clinical forms of leprosy. Further studies will be important for confirming the susceptibility to leprosy per $s e$ in relation to the HLA-DRB1*14 and HLA-DRB1*16 alleles and the significantly lower frequency of HLADRB1*11 in LPs. The racial diversity of the population of MA should be taken into account in such studies.

\section{ACKNOWLEDGEMENTS}

To patients and healthy controls that took part on this study, to the Epidemiology Group of Communicable Disease of the nursing course, and to the HUUFMA. 


\section{REFERENCES}

Alves C, Meyer I, Toralles MBP, Santiago MB 2006. Complexo principal de histocompatibilidade: sua participação na patogênese das doenças reumáticas autoimunes. Rev Bras Promoc Saude 19: 155-163.

Alves C, Meyer I, Vieira N, Toralles MB 2005. Associação do sistema de histocompatibilidade humano (HLA) com doenças endócrinas auto-imunes. Rev Baiana Saude Publica 29: 105-120.

Alves C, Veiga S, Souza T, Toralles MB, Silva-Bacellar AL 2007. Papel del sistema de histocompatibilidad humano en la patogénesis de las enfermedades neurológicas. Rev Neurol 44: 298-302.

Beiguelman B 2002. Genética e hanseníase. Cien Saude Colet 7: $117-128$.

Benjamini E, Coico R, Sunshine G 2002. Imunologia, 4th ed., Guanabara Koogan, Rio de Janeiro, 288 pp.

Dausset J 1958. Iso-leuco-anticorps. Acta Haematol 20: 156-166.

Donadi E 2000. Como entender a nomenclatura e os mecanismos de associação entre antígenos e os alelos de histocompatibilidade com as doenças. Medicina (Ribeirão Preto) 33: 7-18.

Excoffier L, Laval G, Schneider S 2005. Arlequin ver. 3.0: an integrated software package for population genetics data analysis. Evol Bioinform Online 1: 47-50.

Francheschi DS, Sacramento WS, Mazini PS 2009. Hanseníase no mundo moderno: o que sabemos sobre a influência genética do hospedeiro no seu controle? Arq Med 23: 159-165.

Gorodezky C, Alaez C, Munguia A, Cruz R, Vazquez A, Camacho A, Flores S, Rodriguez M, Rodriguez O 2004. Molecular mechanisms of MHC linked susceptibility in leprosy: towards the development of synthetic vaccines. Tuberculosis 84: 1-2.

Goulart LR, Goulart IM 2009. Leprosy pathogenetic backgroud: a review and lessons from other mycobacterial diseases. Arch Dermatol Res 301: 127-137.

Hegazy AA, Abdel-Hamid IA, Ahmed el-SF, Hammad SM, Hawas SA 2002. Leprosy in a high-prevalence Egyptian village: epidemiology and risk factors. Int J Dermatol 41: 681-686.

Hsieh NK, Chu CC, Lee NS, Lee HL, Lin M 2010. Association of HLA-DRB $1 * 0405$ with resistance to multibacillary leprosy in Taiwanese. Hum Immunol 71: 712-716.

Jambeiro JES 2008. Avaliação da neurólise ulnar na neuropatia hansênica. Acta Ortop Bras 16: 207-213.

Joko S, Numaga J, Kawashima H, Namisato M, Maeda H 2000. Human leukocyte antigens in forms of leprosy among Japanese patients. Int J Lepr Other Mycobact Dis 68: 49-56.

Klein J, Sato A 2000. The HLA system. First of two parts. $N$ Engl J Med 7: 702-709.

Lavado-Valenzuela R, Bravo JM, Junqueira-Kipnis AP, Ramos de Souza M, Moreno C, Alonso A, Liberman-Kipnis T, da Silva WD, Cabalero A 2011. Distribution of the HLA class II frequency alleles in patients with leprosy from the mid-west of Brazil. Int $J$ Immun 38: 255-258.
Mira MT 2006. Genetic host resistance and susceptibility to leprosy. Microb Infect 8: 1124-1131.

Monte SJH, Neto JMM, Rampim GF, Shulzenco N, Morgum A, Gerbase-Delima M 2004. Polimorfismo do sistema HLA em uma amostra de mestiços da população de Teresina, Piauí. Rev Assoc Med Bras 50: 422-426.

Moraes MO, Cardoso CC, Vanderborght PR, Pacheco AG 2006. Genetics of host response in leprosy. Lep Reviews 7: 189-202.

Motta PM, Cech N, Fontan C, Giménez MF, Lodeiro N, Marinic K, Molinari ML, Sotelo MG, Habegger SA 2007. Role of HLA-DR and HLA-DQ alleles in multibacillary leprosy and paucibacillary leprosy in the province of Chaco (Argentina). Enferm Infecc Microbiol Clin 25: 627-631.

MS/FNS - Ministério da Saúde/Fundação Nacional da Saúde 1994. Guia de controle da hanseníase, 2nd ed., MS/FNS, Brasília, 156 pp.

Prevedello FC, Mira MT 2007. Hanseníase: uma doença genética? An bras Dermatol 2: 451-459.

Ridley DS, Jopling WH 1966. Classification of leprosy according to immunity. A five group system. Int J Lepr 34: 255-273.

Silva SA, Mazini PS, Reis PG, Sell AM, Tsuneto LT, Peixoto PR 2009. HLA-DR and HLA-DQ alleles in patients from the south of Brazil: markers for leprosy susceptibility and resistance. $B M C$ Infect Dis 9: 134.

Singh M, Balamurugan A, Katoch K 2007. Immunogenetics of mycobacterial infections in the North Indian population. Tissue Antigens 69 (Suppl. 1): 228-230.

Souza VNB, Pereira AC 2007. Genética humana na susceptibilidade à hanseníase. Hansen Int 32: 81-93.

Vanderborght PR, Pacheco AG, Moraes ME 2007. HLA-DRB1*04 and DRB1*10 are associated with resistance and susceptibility, respectively, in Brazilian and Vietnamese leprosy patients. Genes Immun 8: 320-324.

Virmond MCL 2008. Alguns apontamentos sobre a história da prevenção de incapacidades e reabilitação em hanseníase no Brasil. Hansen Int 33 (Suppl.1): 13-18.

Visentainer JE, Tsuneto LT, Serra MF, Peixoto PR, Petzl-Erler ML 1997. Associação de hanseníase com HLA-DR2 em uma população brasileira do sul. Braz J Med Biol Res 30: 51-59.

Walter SL, Lockwood DN 2007. Leprosy. Clin Dermatol 25: 165.

WHO - World Health Organization 2009. Enhanced global strategy for further reducing the disease burden due to leprosy: (plan period: 2011-2015), WHO/Regional Office for South-East Asia, New Delhi, 38 pp.

WHO - World Health Organization 2010. Global leprosy situation 2010. Wkly Epidemiol Rec 85: 337-348.

WHO - World Health Organization 2011. Leprosy update. Wkly Epidemiol Rec 36: 389-400.

Zhang F, Liu H, Chen S, Wang C, Zhu C, Zhang L 2009. Evidence for an association of $H L A-D R B 1^{*} 15$ and $D R B 1^{*} 09$ with leprosy and the impact of $D R B 1^{*} 09$ on disease onset in a Chinese Han population. BMC Med Genet 10: 133. 